Voix et Images

\title{
La littérature québécoise pour la jeunesse : acquis et perspectives
}

\section{Chaké Minassian}

Volume 8, numéro 2, hiver 1983

Marie-Claire Blais

URI : https://id.erudit.org/iderudit/200390ar

DOI : https://doi.org/10.7202/200390ar

Aller au sommaire du numéro

Éditeur(s)

Les Presses de l’Université du Québec

ISSN

0318-9201 (imprimé)

1705-933X (numérique)

Découvrir la revue

Citer cet article

Minassian, C. (1983). La littérature québécoise pour la jeunesse : acquis et perspectives. Voix et Images, 8(2), 357-359. https://doi.org/10.7202/200390ar d'utilisation que vous pouvez consulter en ligne.

https://apropos.erudit.org/fr/usagers/politique-dutilisation/ 


\title{
La littérature québécoise pour la jeunsese: acquis et perspectives
}

\author{
par Chaké Minassian. Université du Québec à Montréal
}

- La littérature québécoise pour la jeunesse? Connais pas!

Il y a à peine une dizaine d'années, telle aurait été la réaction des \& gens du milieun interrogés.

Mais que de chemin parcouru depuis 1972, date a laquelle $\propto$ Communication-Jeunesse» organisait à Montréal, au Pavillon Lafontaine de I'U.O.A.M.. son colloque consacré aux problèmes de la production québécoise dans le domaine de la littérature pour la jeunesse!

Auteurs, illustrateurs, éditeurs, libraires, bibliothécaires, enseignants et autres personnes intéressées avaient tenté alors de diagnostiquer les racines du mal qui avait pour nom méconnaissance; et les effets secondaires désastreux qui s'enchaînaient. selon la formule devenue classique de Paule Daveluy:

"Les livres ne se vendent pas parce qu'on ne les trouve pas en librairie; on ne les trouve pas en librairie parce que le public ne les demande pas; le public ne les demande pas parce qu'il ne les voit pas à l'étalage et que la publicité $n$ 'a pas éveillé sa curiosité: il n'y a pas de publicité parce que l'éditeur n'a pas d'argent pour le faire; l'éditeur n'a pas d'argent parce que les livres ne se vendent pas..."

L'un des grands moments de ce colloque, maintenant que j'y pense avec un recul suffisant, avait assurément été le lancement du livre de Louise Lemieux. Pleins Feux sur la littérature de jeunesse au Canada francais', qui révélait l'existence d'une production québécoise quantitativement fort respectable même si l'on pouvait considérer à juste titre que les contenus de la plupart des livres les plus anciens ne correspondaient plus aux intérèts des enfants de l'ère de la télévision.

Cette dernière vérité serait immanquablement suivie de son corollaire: il fallait "faire quelque chosen, d'abord pour renouveler les contenus de cette littérature si l'on désirait $y$ intéresser le public des jeunes lecteurs québécois 
contemporains, ensute pour prendre les mesures pratiques indispensables afin de permettre que la nouvelle production sorte de sa "clandestinité" et d'empêcher qu'elle croupısse sous la poussière, dans les entrepôts des éditeurs ou sous le comptorr des libraires. comme cela avait été le cas jusqu'alors.

C'est ainsi que nous avons assisté à une prise de conscience graduelle. gràce à l'action superposée, systématique et finalement contagieuse d'un certain nombre de groupes et d'organismes intéressés.

Ainsi, "Communication-Jeunesse», initiatrice du mouvement, multiplia les démarches pratiques et les publications d'information avec la revue «Lurelu», le catalogue sélection a 100 livres a nous, 100 livres pour nous* largement diffusé (il en est à sa quatrième édition), les campagnes publicitaires annuelles pour la promotion des livres, l'organisation des rencontres entre auteurs et élèves de diverses écoles de la province, l'organisation de plusieurs colloques et expositions, animation d'ateliers. etc.

Le Département d'études littéraires de I'U.Q.A.M., novateur, offrit un cours sur "La littérature enfantine de langue française dans le cadre duquel une attention particulière fut accordée à l'examen des difficultés matérielles entourant la production québécoise, a l'analyse des couvres de nos auteurs et à la pratique de la création de textes selon des objectifs visés. La majorité des centaines d'étudiants (enseignants et futurs enseignants pour la plupart). ayant été sensibilisés à l'importance de ce qui était en jeu, se transformèrent en autant de propagateurs de la «bonne parolex, dans leurs milieux respectifs.

L'A.C.A.L.J. (Association canadienne pour l'avancement de la littérature de jeunesse) créée en 1977 après le colloque sur a Le livre dans la vie de l'enfant „, à l'Université de Sherbrooke, publie depuis novembre 1978 la revue Des livres et des jeunes avec, dans chacun de ses numéros, des analyses et des articles de fond consacrés à un aspect particulier de cette littérature globalement prise: mais aussi avec de courtes présentations et évaluations des livres québécois, ce qui contribue à leur promotion.

D'année en année, les subventions officielles se firent plus substantielles. Les ministères concernés s'y intéressèrent de plus près. Les éditeurs prirent courage. Les libraires contribuèrent à mettre en évidence les nouvelles parutions. Les bibliothécaires organisèrent al'heure du contem, etc. etc.

Tout cela me permet d'affirmer avec satisfaction, que la situation déplorable qui prévalait avant 1972, n'existe plus. II y a eu amélioration sous tous les chapitres de la littérature québécoise pour la jeunesse : la quantité des livres a augmenté: l'organisation de leur promotion n'est plus un vain mot; de plus en plus d'éditeurs s'intéressent à cette expérience et se sentent disposés à assumer l'effort pécunier correspondant; de plus en plus de jeunes auteurs et illustrateurs se lancent dans l'aventure, motivés, prêts à fournir l'effort d'imagination nécessaire pour faire face à l'accroissement de la concurrence.

Bref, la période des vaches grasses semble succéder à celle des vaches maigres, au grand bénéfice des jeunes lecteurs. La qualité des livres s'est 
grandement améliorée, tant du point de vue de l'aspect matériel que de celui des contenus et surtout des illustrations.

Cela signıfie-t-il, pour autant, que tout soit pour le mieux dans le meilleur des mondes?

Oui et non. Oui, parce que tous les espoirs nous sont désormais permis.

Non, parce qu ii nous reste encore à accomplir un important effort d'ordre psychologique dans le domaine de la critique.

Je m'explique. Jusqu'à présent, les difficultés d’ordre matériel entourant cette production étaient tellement énormes et insurmontables, que nous avions tendance à saluer avec ravissement l'apparition même de tout livre. Nous n'avions pas tellement tort, car effectivement cela relevait presque du miracle.

Mais fort heureusement, la situation n'est plus la même. Par conséquent. si nous désirons voir se poursuivre dans le sens positif l'évolution entamée, il nous faudrait, maintenir, réviser notre tendance à tout applaudir, développer une attitude plus exigeante, faire place à une critique constructive débarrassée des considérations étrangères à une évaluation intrinsèque des livres.

La littérature québécoise pour la jeunesse a franchi avec succès l'étape de son enfance en l'espace d'une dizaine d'années; elle vit actuellement son adolescence avec des hauts et des bas. Je souhaite qu'elle puisse l'achever avec un minimum de complications dues aux excès de gâteries, afin qu'elle puisse aborder, la tête haute, l'étape de la maturité compétitive à laquelle elle a droit.

En l'espace de dix ans, elle est parvenue à se débarrasser du sentiment de condescendance dont on l'entourait jusqu'alors. Le mérite en revient avant tout aux auteurs et aux illustrateurs eux-mèmes, a leurs tentatives de renouvellement.

Maintenant, leur quvre est prise en considération: l'on semble mème disposé à lui réserver une place appropriée dans la production littéraire globale.

La transformation de cette disposition en habitude définitive, dans les années à venir, dépendra en grande partie de l'idèe que les créateurs euxmèmes se feront de l'importance de leur rôle. Leur propre exigence à l'ègard d'eux-mêmes sera la meilleure garante de l'amélioration constante de cette production.

1. Éditions Leméac, Montréal 1972, $337 \mathrm{p}$. 